\title{
Extensions of Lighthill's acoustic analogy with application to computational aeroacoustics
}

\author{
By C. L. Morfey and M. C. M. Wright \\ Institute of Sound and Vibration Research, University of Southampton, \\ Southampton SO17 1BJ, UK
}

Two extensions to Lighthill's aeroacoustic analogy are presented. First, equivalent sources due to initial conditions are derived that supplement those due to boundary conditions, as given by Ffowcs Williams \& Hawkings [Phil. Trans. Roy. Soc. A264, 321-342 (1969)]. The resulting exact inhomogeneous wave equation is then reformulated with pressure rather than density as the wave variable, and the right-hand side is rearranged using the energy equation with no additional assumptions. A number of source terms emerge that are related to sound generation (or scattering) by entropy inhomogeneities, thermal dissipation, and viscous dissipation.

[to be continued]

Keywords: Aeroacoustics, Initial Value Problem, Green's Functions, Thermoacoustics

\section{Introduction}

The idea of replacing a region of unsteady fluid flow by a distribution of equivalent sources that drive linear perturbations to a base flow has been extremely useful in the field of acoustics. Rayleigh (1894) used equivalent sources to describe scattering of sound in a non-uniform unbounded medium. Lighthill (1952) used the same idea to develop his acoustic analogy in which the equations of fluid motion, expressing conservation of mass and momentum, are rearranged into a linear wave equation with nonlinear forcing terms. In both cases the 'base flow' is a uniform fluid at rest. Provided the forcing terms can be estimated independently of the far-field radiation, Lighthill's equation can be said to describe the nonlinear generation of sound by unsteady flows.

Subsequent extensions and variations of the acoustic analogy include:

1. The addition of equivalent source terms to allow for boundaries in flows that occupy a finite region (Curle 1955, Ffowcs Williams \& Hawkings 1969).

2. Various rearrangements of the source terms to highlight physical processes, often accompanied by a change of wave variable, such as unsteady pressure $p$ (Morfey 1973; Lilley 1974, 1996), the quantity $p+\frac{1}{3} \rho u^{2}$ (Ffowcs Williams 1969 , Kambe 1984), stagnation enthalpy $h+\frac{1}{2} u^{2}$ (Howe 1975), $\left(P / P_{0}\right)^{1 / \gamma}-1$ (Goldstein 2001) etc.

3. The use of a different base flow (Lilley 1974, Goldstein 2003) to match the characteristics of a particular situation, usually jet flow. Howe (1998) and 
Goldstein $(2002,2005)$ discuss a number of such extensions to the original concept of Lighthill (1952).

In this paper we provide two further extensions, one in the first category and one in the second. Both extensions involve use of the energy conservation equation, in contrast to Lighthill (1952) and Ffowcs Williams \& Hawkings (1969) who based their development entirely on mass and momentum conservation. Although several authors have subsequently introduced the energy equation in order to expand the source terms in Lighthill's analogy (Lilley 1974, 1996; Morfey 1973, 1976; Obermeier 1975, 1985; Kempton 1976, $\S \S 6,7$; Kambe \& Minota 1983, appendix A), these formulations are restricted to flows without boundaries. Here we extend the Ffowcs Williams \& Hawkings (1969) treatment of bounded flows (referred to as FWH in what follows) by showing how use of the energy equation leads to a significant reinterpretation of the surface sources in that theory. Other distinctive features of the present work are the use of $p$ as the wave variable, and the use of generalized functions to represent initial conditions as equivalent volume sources in the same way that FWH represents boundary conditions as equivalent surface sources. As with the surface sources, use of the energy equation leads to decisive advantages in formulating the source terms. The base flow is a uniform ideal fluid at rest, but viscous stresses and heat conduction are allowed for in the governing equations, and no restriction is placed on the fluid's equation of state.

The structure of the paper is as follows. [to be continued]

\section{Notation and definitions}

Let $\mathcal{S}$ be a moving closed surface in three dimensions that separates region $\mathcal{V}^{\prime}$ from an adjacent region $\mathcal{V}$, as illustrated in figure 1 . The idea is that $\mathcal{V}^{\prime}$ may contain solid boundaries; alternatively information on the flow in $\mathcal{V}^{\prime}$ may be inaccessible. In either case the aim of the acoustic analogy formulation is to describe the fluctuating pressure or density field in $\mathcal{V}$; no interest attaches to the field in $\mathcal{V}^{\prime}$. Any acoustic influence of $\mathcal{V}^{\prime}$ will be accounted for by equivalent sources on $\mathcal{S}$, and no use will be made of the equations of fluid motion within $\mathcal{V}^{\prime}$. Likewise no use will be made of information for $t<0$; the acoustic influence of events prior to $t=0$ will be accounted for by impulsive sources at $t=0$, distributed throughout region $\mathcal{V}$. Let $f(\mathbf{x}, t)$ be a continuous indicator function such that $f<0$ in $\mathcal{V}^{\prime}, f>0$ in $\mathcal{V}$, and let $|\nabla f|=1$ on $\mathcal{S}$. Smoothness of $\mathcal{S}$ is assumed, so that $\nabla f$ is single-valued $\dagger$. Let $n$ be a local normal co-ordinate, defined for points near $\mathcal{S}$ by $n=f$; then $\partial / \partial n$ evaluated on $\mathcal{S}$ is the gradient operator normal to $\mathcal{S}$, in the direction from $\mathcal{V}^{\prime}$ to $\mathcal{V}$. Define the spatial and temporal Heaviside functions

$$
\begin{aligned}
& \mathrm{H}(n)= \begin{cases}1 & \text { in } \mathcal{V} \text { and on } \mathcal{S} \\
0 & \text { in excluded region } \mathcal{V}^{\prime}\end{cases} \\
& \Theta(t)= \begin{cases}1 & \text { for } t \geq 0 \\
0 & \text { for } t<0\end{cases}
\end{aligned}
$$

$\dagger$ An extension of this description to cusped surfaces, such as a sharp-edged airfoil, has been presented by Farassat \& Myers (1990).

Article submitted to Royal Society 
(a)

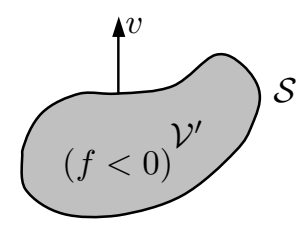

$\mathcal{V}$

$(f>0)$ (b)

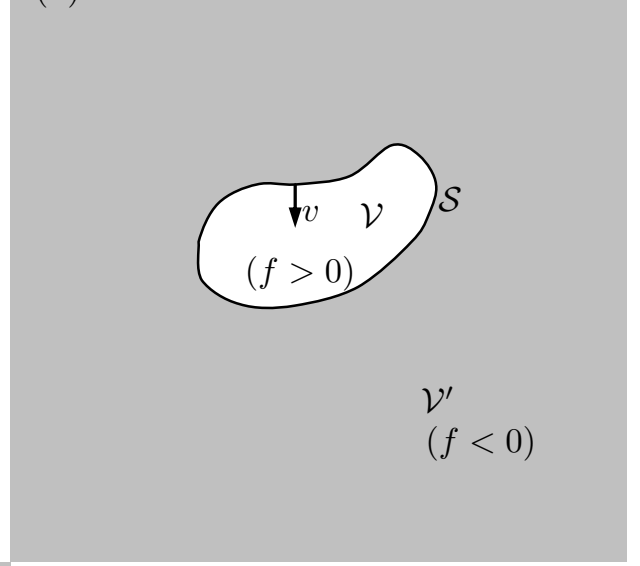

(d)

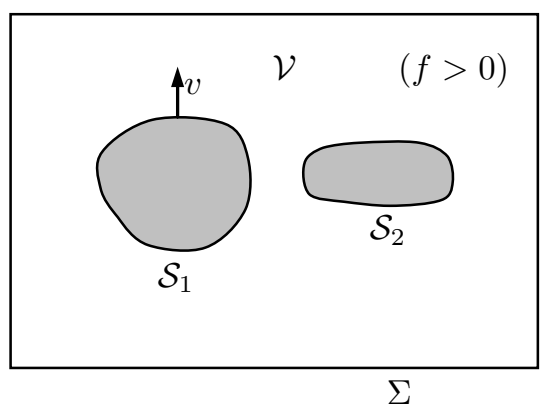

Figure 1. Schematic diagram showing the complementary regions $\mathcal{V}^{\prime}$ (about which no knowledge is available) and $\mathcal{V}$, and the interface $\mathcal{S}$ between them. In region $\mathcal{V}$ either the equations of fluid motion apply for $t>0$, as in $\S \S 3 \&$ ??; or (alternatively) a scalar wave equation is valid for $t>0$. Region $\mathcal{V}$ may be exterior to $\mathcal{S}$ as in (a), or interior as in (b). More generally, (c) shows that the excluded region $\mathcal{V}^{\prime}$ may be multiply connected. In all these cases, $\mathcal{V} \cup \mathcal{V}^{\prime}$ fills the entire space. A further option, shown in (d), is to have $\mathcal{V} \cup \mathcal{V}^{\prime}$ surrounded by a closed surface $\Sigma$, that lies in a region of linear acoustic disturbances to the reference state $\left(\rho_{0}, c_{0}\right)$, and represents an acoustically absorbing or scattering boundary. Case (a) can be regarded as a limiting case of (d) in which $\Sigma$ becomes a sphere of infinite radius and the Sommerfeld radiation condition is applied.

which henceforth will be written without their arguments. From the definition of the Heaviside function we have

$$
\frac{\partial \Theta}{\partial t}=\delta(t), \quad \frac{\partial \Theta}{\partial x_{i}}=0, \quad \frac{\partial \mathrm{H}}{\partial n}=\delta(n), \quad \frac{\partial \mathrm{H}}{\partial x_{i}}=\hat{n}_{i} \delta(n)
$$

where $\delta(\cdot)$ is the Dirac delta function and $\hat{n}_{i}=\partial n / \partial x_{i}$, the unit normal to $\mathcal{S}$. The time derivative of $\mathrm{H}$ is found by noting that $\mathrm{H}$ is constant in a reference frame 
moving with the surface, so that

$$
\frac{\partial \mathrm{H}}{\partial t}=-v_{i} \frac{\partial \mathrm{H}}{\partial x_{i}}=-v_{i} \hat{n}_{i} \delta(n)=-v \delta(n) .
$$

Here $v_{i}=v \hat{n}_{i}$, with $v$ the normal velocity of the surface $\mathcal{S}$ directed into $\mathcal{V}$. The material derivative of $\mathrm{H}$ is given by

$$
\frac{\mathrm{DH}}{\mathrm{D} t}=\left(u_{i}-v_{i}\right) \hat{n}_{i} \delta(n) .
$$

where $\mathrm{D} / \mathrm{D} t=\left(\partial / \partial t+u_{i} \partial / \partial x_{i}\right)$.

In what follows, a line over any variable or quantity means that it is multiplied by $\Theta \mathrm{H}$, thus windowing it in space and time. For consistency, generalized functions are written at the end of a product, with spatial generalized functions preceding temporal ones. Using the relations given above we can find the result of commuting the windowing operation with differentiation with respect to space and time respectively:

$$
\frac{\overline{\partial \xi}}{\partial t}-\frac{\partial \bar{\xi}}{\partial t}=\xi\left[v_{i} \hat{n}_{i} \delta(n) \Theta-\mathrm{H} \delta(t)\right], \quad \frac{\overline{\partial \xi}}{\partial x_{i}}-\frac{\partial \bar{\xi}}{\partial x_{i}}=-\xi \hat{n}_{i} \delta(n) \Theta .
$$

The identity

$$
\frac{\partial \xi}{\partial t} \equiv \frac{\mathrm{D} \xi}{\mathrm{D} t}-\frac{\partial}{\partial x_{i}}\left(\xi u_{i}\right)+\xi \Delta,
$$

also holds wherever $u_{i}$ is defined; here $\Delta=\partial u_{i} / \partial x_{i}$ is the dilatation rate. An important, but lengthy, derivation of the second time derivative of an arbitrary windowed variable is given in Appendix A.

\section{Initial-boundary value formulations for aeroacoustics}

As a starting point for deriving a generalized statement of Lighthill's acoustic analogy that incorporates both initial and boundary conditions, we take the windowed equations of motion for a fluid occupying region $\mathcal{V}$. Conservation of mass and momentum are expressed by

$$
\overline{\frac{\partial\left(\rho-\rho_{0}\right)}{\partial t}}+\overline{\frac{\partial}{\partial x_{i}}\left(\rho u_{i}\right)}=0, \quad \overline{\frac{\partial}{\partial t}\left(\rho u_{i}\right)}+\overline{\frac{\partial}{\partial x_{j}}\left(\rho u_{i} u_{j}+p_{i j}\right)}=\overline{G_{i}} .
$$

Here and throughout, subscript 0 denotes the properties of a uniform reference medium, chosen to coincide with the actual flow in the acoustic far field. Without loss of generality, we choose a frame of reference that makes the fluid velocity zero at infinity. In (3.1), $\rho$ denotes fluid density; $u_{i}$ is the fluid velocity in the $x_{i}$ direction; $p_{i j}=P_{i j}-P_{0} \delta_{i j}$ where $P$ is absolute pressure and $P_{i j}$ is the compressive stress in the fluid; $\delta_{i j}$ is the Kronecker delta, and $G_{i}$ is an applied body force per unit volume. The quantities $\left(\rho-\rho_{0}\right), \rho u_{i}, \rho u_{i} u_{j}+p_{i j}, G_{i}$ in (3.1) all vanish in the far-field region.

We wish to obtain an acoustic analogy in terms of windowed variables. Applying equations (2.6) to the conservation equations (3.1) produces additional terms on the right hand side:

$$
\frac{\partial\left(\overline{\rho-\rho_{0}}\right)}{\partial t}+\frac{\partial}{\partial x_{i}}\left(\overline{\rho u_{i}}\right)=\left(\rho-\rho_{0}\right) \mathrm{H} \delta(t)+\left[\rho u_{i}-\left(\rho-\rho_{0}\right) v_{i}\right] \hat{n}_{i} \delta(n) \Theta
$$


and

$$
\frac{\partial}{\partial t}\left(\overline{\rho u_{i}}\right)+\frac{\partial}{\partial x_{j}}\left(\overline{\rho u_{i} u_{j}+p_{i j}}\right)=\overline{G_{i}}+\rho u_{i} \mathrm{H} \delta(t)+\left[\rho u_{i}\left(u_{j}-v_{j}\right)+p_{i j}\right] \hat{n}_{j} \delta(n) \Theta .
$$

By eliminating $\overline{\rho u_{i}}$ from (3.2) \& (3.3) we obtain an expression for the second time derivative of $\frac{\rho-\rho_{0}}{}$, the windowed density perturbation, that is valid for all $\left(x_{i}, t\right)$ :

$$
\begin{aligned}
\frac{\partial^{2}\left(\overline{\rho-\rho_{0}}\right)}{\partial t^{2}}= & \frac{\partial}{\partial t}\left[\left(\rho-\rho_{0}\right) \mathrm{H} \delta(t)\right]-\frac{\partial}{\partial x_{i}}\left[\rho u_{i} \mathrm{H} \delta(t)\right] \\
& +\frac{\partial}{\partial t}\left\{\left[\rho u_{i}-\left(\rho-\rho_{0}\right) v_{i}\right] \hat{n}_{i} \delta(n) \Theta\right\} \\
& -\frac{\partial}{\partial x_{i}}\left\{\left[\rho u_{i}\left(u_{j}-v_{j}\right)+p_{i j}\right] \hat{n}_{j} \delta(n) \Theta\right\} \\
& -\frac{\partial \overline{G_{i}}}{\partial x_{i}}+\frac{\partial^{2}}{\partial x_{i} \partial x_{j}}\left(\overline{\rho u_{i} u_{j}+p_{i j}}\right) .
\end{aligned}
$$

(a) Density form of the acoustic analogy

Subtracting $\nabla^{2}\left(\overline{\rho-\rho_{0}}\right)$ from (3.4) leads directly to

$$
\begin{aligned}
\left(\frac{1}{c_{0}^{2}} \frac{\partial^{2}}{\partial t^{2}}-\nabla^{2}\right)\left[c_{0}^{2}\left(\overline{\rho-\rho_{0}}\right)\right]= & \frac{\partial}{\partial t}\left[\left(\rho-\rho_{0}\right) \mathrm{H} \delta(t)\right]-\frac{\partial}{\partial x_{i}}\left[\rho u_{i} \mathrm{H} \delta(t)\right] \\
& +\frac{\partial}{\partial t}\left[J_{i} \hat{n}_{i} \delta(n) \Theta\right]-\frac{\partial}{\partial x_{i}}\left[L_{i j} \hat{n}_{j} \delta(n) \Theta\right] \\
& -\frac{\partial \overline{G_{i}}}{\partial x_{i}}+\frac{\partial^{2} \overline{T_{i j}}}{\partial x_{i} \partial x_{j}} .
\end{aligned}
$$

Symbols $T_{i j}, J_{i}$ and $L_{i j}$ on the right of (3.5) stand for the Lighthill stress tensor

$$
T_{i j}=\rho u_{i} u_{j}+p_{i j}-c_{0}^{2}\left(\rho-\rho_{0}\right) \delta_{i j},
$$

the surface mass flux vector

$$
J_{i}=\rho u_{i}-\left(\rho-\rho_{0}\right) v_{i}=\rho\left(u_{i}-v_{i}\right)+\rho_{0} v_{i},
$$

and the surface momentum flux tensor

$$
L_{i j}=\rho u_{i}\left(u_{j}-v_{j}\right)+p_{i j} .
$$

The sources on the right hand side of (3.5) can be interpreted as follows:

1. The first two terms represent the impulsive addition of mass and momentum needed to start the flow from its initial reference state.

2. The second line contains the usual FWH surface monopoles and dipoles, windowed by $\Theta$.

3. Volume source terms appear in the third line, with the body force $G_{i}$ and the Lighthill stress tensor $T_{i j}$ windowed spatially and temporally by $\Theta \mathrm{H}$. 
Equation (3.5) without the initial-value source terms is the standard FWH equation and has been widely used in computational aeroacoustics, where it provides a means of extrapolation from the simulation domain to the acoustic far field $\dagger$. However, in this context (3.5) is not well suited to applications involving heated flows, or flows in which mixing occurs between different fluids (Shur et al. 2005, Spalart et al. 2007). The reason is that the surface monopole and dipole distributions, $J_{i} \hat{n}_{i}$ and $L_{i j} \hat{n}_{j}$, depend on the local density; so fluctuations in these quantities occur when local hot spots, or regions of different fluid composition, are advected across the control surface $\mathcal{S}$. Such advection has little to do with sound radiation.

It is important to recognize that (3.5) remains valid for heated and inhomogeneous flows; the physically unrealistic surface sources described above are cancelled by terms in the quadrupole distribution $\overline{T_{i j}}$. What this means is that neglect of the volume quadrupoles $\overline{T_{i j}}$ is not justified under such conditions. For wave extrapolation purposes, therefore, there is a strong incentive to find alternative formulations that cope better with advected density disturbances.

\section{(b) Density-substituted forms of the acoustic analogy}

Two formulations of the extended Lighthill analogy are presented below in which the local density is absent, both form the surface monopole and dipole distributions, and from the initial-value source terms. The first version applies to an arbitrary fluid, and the second version applies to a particular class of fluids that includes perfect gases.

Both version begin from the $\partial^{2}\left(\overline{\rho-\rho_{0}}\right) / \partial t^{2}$ expression $(3.4)$, and use the kinematic relation (A 4 ) for the second time derivative of an arbitrary windowed variable, $\bar{\xi}$, to replace $\rho$ by a new variable $\rho^{+}$related to the local pressure. By defining

$$
\xi=\rho-\rho^{+}
$$

and subtracting $\partial^{2} \bar{\xi} / \partial t^{2}$ from $\partial^{2}\left(\overline{\rho-\rho_{0}}\right) / \partial t^{2}$, an equation for $\partial^{2}\left(\overline{\rho^{+}-\rho_{0}}\right) / \partial t^{2}$ is obtained that exhibits the properties mentioned above:

$$
\begin{aligned}
\frac{\partial^{2}\left(\overline{\rho^{+}-\rho_{0}}\right)}{\partial t^{2}}= & \frac{\partial}{\partial t}\left[\left(\rho^{+}-\rho_{0}\right) \mathrm{H} \delta(t)\right]-\frac{\partial}{\partial x_{i}}\left[\rho^{+} u_{i} \mathrm{H} \delta(t)\right] \\
& \frac{\partial}{\partial t}\left[J_{i}^{+} \hat{n}_{i} \delta(n) \Theta\right]-\frac{\partial}{\partial x_{i}}\left[L_{i j}^{+} \hat{n}_{j} \delta(n) \Theta\right] \\
& +\frac{\partial \overline{Q^{+}}}{\partial t}-\frac{\partial}{\partial x_{i}}\left[\overline{Q^{+} u_{i}-\left(\rho-\rho^{+}\right) \frac{\mathrm{D} u_{i}}{\mathrm{D} t}+G_{i}}\right] \\
& +\frac{\partial^{2}}{\partial x_{i} \partial x_{j}}\left(\overline{\rho^{+} u_{i} u_{j}+p_{i j}}\right) .
\end{aligned}
$$

Here $J_{i}^{+}$and $L_{i j}$, are defined in the same way as $J_{i}$ and $L_{i j}$ with $\rho$ replaced by $\rho^{+}$, and $Q^{+}$is defined as

$$
Q^{+}=-\left[\frac{\mathrm{D}\left(\rho-\rho^{+}\right)}{\mathrm{D} t}+\left(\rho-\rho^{+}\right) \Delta\right]=\frac{\mathrm{D} \rho^{+}}{\mathrm{D} t}+\rho^{+} \Delta
$$

where the second version follows from mass conservation.

$\dagger$ See $\S ?$ ? below. 
A 'generic acoustic analogy' can then be written as

$$
\begin{aligned}
\left(\frac{1}{c_{0}^{2}} \frac{\partial^{2}}{\partial t^{2}}-\nabla^{2}\right)\left[c_{0}^{2}\left(\overline{\rho^{+}-\rho_{0}}\right)\right]= & \frac{\partial}{\partial t}\left[\left(\rho^{+}-\rho_{0}\right) \mathrm{H} \delta(t)\right]-\frac{\partial}{\partial x_{i}}\left[\rho^{+} u_{i} \mathrm{H} \delta(t)\right] \\
& \frac{\partial}{\partial t}\left[J_{i}^{+} \hat{n}_{i} \delta(n) \Theta\right]-\frac{\partial}{\partial x_{i}}\left[L_{i j}^{+} \hat{n}_{j} \delta(n) \Theta\right] \\
& +\frac{\partial \overline{Q^{+}}}{\partial t}-\frac{\partial}{\partial x_{i}}\left(\overline{Q^{+} u_{i}+\frac{\rho^{+}}{\rho} G_{i}+\frac{\rho-\rho^{+}}{\rho} \frac{\partial p_{i j}}{\partial x_{j}}}\right) \\
& +\frac{\partial^{2} \overline{T_{i j}^{+}}}{\partial x_{i} \partial x_{j}},
\end{aligned}
$$

where the penultimate term has been obtained by writing the equation of conservation of momentum in the form

$$
\frac{\mathrm{D} u_{i}}{\mathrm{D} t}=\frac{G_{i}}{\rho}-\frac{1}{\rho} \frac{\partial p_{i j}}{\partial x_{j}},
$$

which is valid throughout $\mathcal{V}$, and where $T_{i j}^{+}$is defined in the same way as $T_{i j}$ with $\rho$ replaced by $\rho^{+}$.

Like (3.4), equation (??) is exact; it applies to bounded domains $(f>0, t>0)$; and no assumption has been made about the fluid equation of state. Its usefulness, as the basis of an acoustic analogy, depends on the term $\partial \overline{Q^{+}} / \partial t$ being sufficiently small that its contribution from any acoustic region can be neglected; we examine this issue next, for two particular choices of the variable $\rho^{+}$.

\section{(c) Determination of $Q^{+}$from the energy equation}

If we choose the substituted density variable $\rho^{+}$as the acoustic density $\rho^{\star}$, defined by

$$
\rho^{\star}=\rho_{0}+c_{0}^{-2} p=\rho_{0}\left(1+K_{0} p\right)
$$

where $K$ is the isentropic compressibility $1 /\left(\rho c^{2}\right)$, then the corresponding value of $Q^{+}$is given by $(3.11)$ as

$$
Q^{\star}=\rho_{0}\left[\Delta+K_{0}\left(p \Delta+\frac{\mathrm{D} p}{\mathrm{D} t}\right)\right] .
$$

From the energy equation for a single-component $\dagger$ viscous heat-conducting fluid, with heat input rate $\dot{q}$ per unit volume, it follows that

$$
\begin{aligned}
-\frac{1}{\rho} \frac{\mathrm{D} \rho}{\mathrm{D} t}+K \frac{\mathrm{D} p}{\mathrm{D} t} & =\frac{\alpha}{\rho c_{p}}\left(\Phi-\frac{\partial q_{i}}{\partial x_{i}}+\dot{q}\right) \\
& =\Delta^{\bullet}
\end{aligned}
$$

where $\Phi$ is the viscous dissipation function

$$
\Phi=\tau_{i j} \frac{\partial u_{i}}{\partial x_{j}},
$$

$\dagger$ For a mixture of two fluids a generalization of (3.15) is given in appendix II of Morfey (1976). 
and $q_{i}$ is the heat flux vector; other symbols are $\alpha$ for the volumetric thermal expansivity and $c_{p}$ for the constant-pressure specific heat. The quantity $\Delta^{\bullet}$ is the difference between the actual dilatation rate and that due to isentropic compression; we therefore refer to $\Delta^{\bullet}$ as the entropic dilatation rate. Alternative version of (3.15) using $\Delta^{\bullet}$ are

$$
\begin{aligned}
Q^{\star} & =\rho_{0}\left(1+K_{0} p\right) \Delta^{\bullet}-\rho_{0}\left(K-K_{0}+K_{0} K p\right) \frac{\mathrm{D} p}{\mathrm{D} t} \\
& =\rho_{0}\left(K_{0} / K\right) \Delta^{\bullet}+\rho_{0}\left(1-K_{0} / K+K_{0} p\right) \Delta
\end{aligned}
$$

It is clear from (3.18) that in a region where the only disturbances are sound waves, $Q^{\star}$ is indeed small. Its inclusion in the acoustic analogy source term, in equation (3.20) below, accounts for thermal attenuation of sound and for nonlinear acoustic phenomena.

The first density-substituted acoustic form of the acoustic analogy is therefore obtained by setting $\rho^{+}=\rho^{\star}$ in (3.12), which gives an equation with acoustic pressure as the wave variable:

$$
\begin{aligned}
\left(\frac{1}{c_{0}^{2}} \frac{\partial^{2}}{\partial t^{2}}-\nabla^{2}\right) \bar{p}= & \frac{1}{c_{0}^{2}} \frac{\partial}{\partial t}[p \mathrm{H} \delta(t)]-\frac{\partial}{\partial x_{i}}\left[\rho^{\star} u_{i} \mathrm{H} \delta(t)\right] \\
& +\frac{\partial}{\partial t}\left[J_{i}^{\star} \hat{n}_{i} \delta(n) \Theta\right]-\frac{\partial}{\partial x_{i}}\left[L_{i j}^{\star} \hat{n}_{j} \delta(n) \Theta\right] \\
& +\frac{\partial \overline{Q^{\star}}}{\partial t}-\frac{\partial}{\partial x_{i}}\left(\overline{Q^{\star} u_{i}+\frac{\rho^{\star}}{\rho} G_{i}+\frac{\rho-\rho^{\star}}{\rho} \frac{\partial p_{i j}}{\partial x_{j}}}\right) \\
& +\frac{\partial^{2} \overline{T_{i j}^{\star}}}{\partial x_{i} \partial x_{j}},
\end{aligned}
$$

where $J_{i}^{\star}, L_{i j}^{\star}$ and $T_{i j}^{\star}$ are $J_{i}^{+}, L_{i j}^{+}$and $T_{i j}^{+}$with $\rho^{+}=\rho^{\star}$, so

$$
T_{i j}^{\star}=\rho^{\star} u_{i} u_{j}+p_{i j}-c_{0}^{2}\left(\rho^{\star}-\rho_{0}\right) \delta_{i j}=\rho^{\star} u_{i} u_{j}-\tau_{i j},
$$

where $\tau_{i j}$ is the viscous stress such that $p_{i j}=p \delta_{i j}-\tau_{i j}$.

The presence of convected density inhomogeneities in the flow will make $\rho \neq$ $\rho^{\star}$, even in a non-conducting fluid. The dipole body force term then depends on fluctuations in the body force per unit mass $G_{i} / \rho$ rather than $G_{i}$, and an extra dipole term appears (the term in $p_{i j}$ on the last line of (3.20)). The $p_{i j}$ term acts like an additional body force applied to the reference medium; it is the generalization to viscous flows of the dipole source term identified in Morfey (1973), Lilley (1974) and Howe (1998).

The second density-substituted form of the acoustic analogy, suggested by Spalart (private communication 2006, Spalart et al. 2007) uses $\rho^{+}=\rho^{\diamond}$ where

$$
\rho^{\diamond}=\rho_{0}\left(1+\frac{p}{P_{0}}\right)^{1 / \gamma}
$$

[why $Q^{\diamond}$ is quiet...]

The corresponding acoustic analogy therefore has the same RHS as (3.20) with $\star$ replaced by $\diamond$ throughout, and LHS

$$
\left(\frac{1}{c_{0}^{2}} \frac{\partial^{2}}{\partial t^{2}}-\nabla^{2}\right) c_{0}^{2}\left(\overline{\rho^{\diamond}-\rho}\right) .
$$


(d) Implications for computational aeroacoustics (CAA)

In CAA, a 2-stage procedure - called direct noise computation in the reviews by Bailly \& Bogey (2004) and Colonius \& Lele (2004) — is used to calculate the far-field sound radiated by a region of turbulent or unsteady flow. An accurate numerical simulation is first performed to capture the unsteady flow in a limited domain $\mathcal{D}$, which is chosen to extend as far into the surrounding region of smaller-amplitude unsteadiness as computational costs allow. Boundary conditions on $\mathcal{D}$ are chosen so as to minimize the reflection of outgoing acoustic waves. The resulting simulation in $\mathcal{D}$ is then extended to the far field by one of several methods that typically involve linearized approximations to the flow equations and are less demanding computationally (Colonius \& Lele 2004).

Since the late 1980s, two popular choices for far-field extension of accurate nearfield simulations have been the analytically-based FWH method and the related Kirchhoff method, both of which rely on the flow outside $\mathcal{D}$ approximating a uniform acoustic medium with small-amplitude disturbances governed by the wave equation. Brentner \& Farassat (1998) have carried out a detailed comparison of the FWH and Kirchhoff methods as applied to transonic rotor noise. By calculating the far-field radiation with $\mathcal{S}$ taken progressively further from the rotor, they were able to show that FWH converged more rapidly with increasing distance. A similar conclusion was reached by Singer et al. (2000) who studied the sound field of a long rigid cylinder in subsonic cross-flow $(M=0.2)$ with a turbulent wake. Since the FWH and Kirchhoff formulations are both exact if all the terms are retained, these differences must be due to the neglected volume terms being different. Specifically, since both studies were for unheated, homogeneous-fluid flows with $\left(\rho-\rho^{\star}\right) / \rho_{0} \sim M^{2}$, they are due to the quadrupole term $\partial^{2} \overline{S_{i j}} / \partial x_{i} \partial x_{j}$ being a weaker source of sound than the windowed quadrupole term $\overline{\partial^{2} S_{i j} / \partial x_{i} \partial x_{j}}$ Since the far-field solution was obtained with the free-field Green's function in both cases and the radiating surface $\mathcal{S}$ was compact with respect to the lower radiated frequencies, weaker radiation is expected from the term in (iv).

For CAA calculations of jet noise, different problems arise with the FWH and Kirchhoff techniques for far-field extrapolation, because jets of practical interest are typically heated (as in aircraft gas turbine exhausts). The $\left(\rho-\rho^{\star}\right)$ terms cannot be neglected, and they decay slowly in the downstream direction. A recent review of CAA results for turbulent jets (Shur et al. 2005) draws attention to this problem, and offers a pragmatic solution: the authors recommend that in the FWH surface terms $\rho$ should be replaced by $\rho^{\star}=\rho_{0}+c_{0}^{-2} p$. This change arises naturally in the EDR formulation and provides evidence that the EDR formulation is better suited to some CAA problems than FWH.

Since initial values are usually ignored in such calculations, as are the volume sources, the equation to be used is, effectively

$$
\left(\frac{1}{c_{0}^{2}} \frac{\partial^{2}}{\partial t^{2}}-\nabla^{2}\right) \bar{p} \approx \frac{\partial}{\partial t}\left[J_{i}^{\star} \hat{n}_{i} \delta(n) \Theta\right]-\frac{\partial}{\partial x_{i}}\left[L_{i j}^{\star} \hat{n}_{j} \delta(n) \Theta\right]
$$

with

$$
J_{i}^{\star}=\rho_{0} u_{i}+c_{0}^{-2} p\left(u_{i}-v_{i}\right) \quad \text { and } \quad L_{i j}^{\star}=\left(\rho_{0}+c_{0}^{-2} p\right) u_{i}\left(u_{j}-v_{j}\right)+p_{i j}
$$


or

$$
\left(\frac{1}{c_{0}^{2}} \frac{\partial^{2}}{\partial t^{2}}-\nabla^{2}\right) c_{o}^{2}\left(\overline{\rho^{\diamond}-\rho_{0}}\right) \approx \frac{\partial}{\partial t}\left[J_{i}^{\diamond} \hat{n}_{i} \delta(n) \Theta\right]-\frac{\partial}{\partial x_{i}}\left[L_{i j}^{\diamond} \hat{n}_{j} \delta(n) \Theta\right]
$$

with the equivalent expressions for $J_{i}^{\diamond}$ and $L_{i j}^{\diamond}$. Spalart et al. (2007) used both equations to calculate the sound radiated from an LES-simulated jet and found the difference to be negligible.

\section{Base-flow formulation}

The Lilley-Goldstein analogy equation may be written in the compact form

$$
\begin{aligned}
\mathcal{L}(\bar{\pi})= & \frac{\tilde{\mathrm{D}}^{2} \bar{\sigma}}{\mathrm{D} t^{2}}+L_{j}\left(\overline{\sigma_{j}}-\frac{\partial \overline{\sigma_{i j}}}{\partial x_{i}}\right) \\
& +\frac{\tilde{\mathrm{D}}^{2}}{\mathrm{D} t^{2}}[\pi \mathrm{H} \delta(t)]+L_{j}\left[(1+\pi) u_{j}^{\prime} \mathrm{H} \delta(t)\right] \\
& +\frac{\tilde{\mathrm{D}}^{2}}{\mathrm{D} t^{2}}\left\{\left[u_{i}^{\prime}+\pi\left(u_{i}-v_{i}\right)\right] \hat{n}_{i} \delta(n) \Theta\right\} \\
& +L_{j}\left\{\left[\tilde{c}^{2} \pi \delta_{i j}+(1+\pi)\left(u_{i}-v_{i}\right) u_{j}^{\prime}\right] \hat{n}_{i} \delta(n) \Theta\right\},
\end{aligned}
$$

where the operators $\mathcal{L}$ and $L_{j}$ are defined in (??) \& (??). The volume source distributions $\sigma$ (monopole), $\sigma_{j}$ (dipole), and $\sigma_{i j}$ (quadrupole) are given by

$$
\begin{aligned}
& \sigma=(1+\pi) \Delta^{\bullet} \\
& \sigma_{j}=-\left(c^{2}\right)^{\prime} \frac{\partial \pi}{\partial x_{j}}+(1+\pi) g_{j}+(1+\pi)\left(u_{j}^{\prime} \Delta^{\bullet}+\frac{1}{\rho} \frac{\partial \pi_{i j}}{\partial x_{i}}\right), \\
& \sigma_{i j}=(1+\pi) u_{i}^{\prime} u_{j}^{\prime} .
\end{aligned}
$$

Also $\tilde{c}\left(x_{2}, x_{3}\right)$ is the base-flow sound speed, related to the base-flow density $\tilde{\rho}\left(x_{2}, x_{3}\right)$ by $\tilde{\rho} \tilde{c}=$ const., and $\left(c^{2}\right)^{\prime}$ is defined by

$$
\left(c^{2}\right)^{\prime}=c^{2}-\tilde{c}^{2}
$$

Note that the base-flow velocity $\tilde{u}\left(x_{2}, x_{3}\right)$ appears in the derivative operator

$$
\frac{\tilde{\mathrm{D}}}{\mathrm{D} t} \equiv \frac{\partial}{\partial t}+\tilde{u} \frac{\partial}{\partial x_{1}}
$$

but is otherwise absent in explicit form from (4.1).

\section{Interpretation of $Q^{\star}$ or $Q^{\diamond}$}

The monopole density $Q^{\star}$ is non-zero in general. However in an ideal fluid its effect is limited to the scattering of sound by sound (nonlinear acoustics), or to scattering in an inhomogeneous medium by variations of compressibility (for example in a bubbly liquid); whereas in real turbulent flows, fluctuations in $Q^{\star}$ also arise from unsteady viscous or thermal dissipation. 
An exact expression for $Q^{\star}$ in perfect-gas flows that is convenient for computational studies follows from (3.16) and (3.19):

$$
Q^{\star}=\frac{\gamma-1}{c_{0}^{2}}\left(\Phi-p \Delta-\frac{\partial q_{i}}{\partial x_{i}}+\dot{q}\right),
$$

where $\gamma$ is the specific-heat ratio.

To interpret $Q^{\star}$ for the general case of an arbitrary fluid, define the excess compressibility $K_{e}$ as

$$
\begin{aligned}
K_{e} & =K-K_{0}-\left(\frac{\partial K}{\partial P}\right)_{s, 0} p \\
& =K-K_{0}+\left(2 \beta_{0}-1\right) K_{0}^{2} p
\end{aligned}
$$

the partial derivative $(\partial K / \partial P)_{s}$ is evaluated holding the specific entropy $s$ constant and $\beta$ is the nonlinearity parameter $c^{-1}(\partial(\rho c) / \partial \rho)_{s}$. Then (3.18) gives

$$
\begin{aligned}
Q^{\star} & =\rho_{0}\left(1+K_{0} p\right)\left[\Delta^{\bullet}-K_{e} \frac{\mathrm{D} p}{\mathrm{D} t}+\left(\beta_{0}-1\right) K_{0}^{2} \frac{\mathrm{D} p^{2}}{\mathrm{D} t}\right]+\rho_{0} K_{0}^{3} p^{2} \frac{\mathrm{D} p}{\mathrm{D} t} \\
& =\rho_{0}\left[\Delta^{\bullet}-K_{e} \frac{\mathrm{D} p}{\mathrm{D} t}+\left(\beta_{0}-1\right) K_{0}^{2} \frac{\mathrm{D} p^{2}}{\mathrm{D} t}\right]\left[1+O\left(K_{0} p\right)\right] .
\end{aligned}
$$

The three terms in the first bracket each have a physical interpretation.

1. The entropic dilatation rate $\Delta^{\bullet}$ is given by the energy equation (3.15). It contains contributions

$$
\begin{array}{ll}
\Delta_{\mu}^{\bullet}=\frac{\alpha}{\rho c_{p}} \Phi & \text { due to viscous dissipation; } \\
\Delta_{\kappa}^{\bullet}=-\frac{\alpha}{\rho c_{p}} \frac{\partial q_{i}}{\partial x_{i}}=\frac{\alpha}{\rho c_{p}} \frac{\partial}{\partial x_{i}}\left(\kappa \frac{\partial T}{\partial x_{i}}\right) & \text { due to heat conductivity } \kappa \\
\Delta_{q}^{\bullet}=\frac{\alpha}{\rho c_{p}} \dot{q} & \text { due to external heat sources. }
\end{array}
$$

In an Euler equation model, only $\Delta_{q}^{\bullet}$ survives.

2. The $-K_{e} \mathrm{D} p / \mathrm{D} t$ term is Rayleigh's monopole scattering term (Rayleigh 1894). It accounts for sound amplification and scattering by bubble clouds in liquids, or by any variation in compressibility of the medium.

3. The nonlinear $\mathrm{D} p^{2} / \mathrm{D} t$ term combines with the quadrupole term in the last line of (3.20) to produce the Westervelt source term of nonlinear acoustics (Hamilton \& Morfey 1997).

\section{(a) Thermoacoustic sources}

The monopole source term $\left(\partial \overline{Q^{\star}} / \partial t\right)$ in (3.20) is analysed further to show how temperature gradients lead to thermoacoustic sources. For unsteady flows with $M^{2} \ll 1$,

$$
\frac{\partial \overline{Q^{\star}}}{\partial t}=\rho_{0} \frac{\partial \overline{\Delta^{\bullet}}}{\partial t}+O\left(M^{2}\right)
$$


Here we have used (5.3), and we are assuming $K_{e} / K_{0}$ is $O\left(M^{2}\right)$; in other words any variations of fluid compressibility due to gradients of entropy or composition are of the same order of magnitude as those due to pressure variationst. We further assume that external heat sources are absent, so that $\dot{q}=0$. Then in flows with $\Delta T / T=O(1)$, the dominant term in $\Delta^{\bullet}$ is due to heat conduction:

$$
\Delta^{\bullet}=-\frac{\alpha}{\rho c_{p}} \frac{\partial q_{i}}{\partial x_{i}}\left[1+O\left(M^{2}\right)\right],
$$

giving (for $\Theta=1$ ) the following expression for $\overline{\Delta^{\bullet}}$ in (5.7):

$$
\begin{aligned}
\Delta^{\bullet} \mathrm{H} & \approx-\frac{\alpha}{\rho c_{p}} \mathrm{H} \frac{\partial q_{i}}{\partial x_{i}} \\
& =\frac{\alpha}{\rho c_{p}} q_{i} \hat{n}_{i} \delta(n)+q_{i} \frac{\partial}{\partial x_{i}}\left(\frac{\alpha}{\rho c_{p}}\right) \mathrm{H}-\frac{\partial}{\partial x_{i}}\left[\frac{\alpha}{\rho c_{p}} q_{i} \mathrm{H}\right] .
\end{aligned}
$$

We shall call these terms $\Delta_{1}^{\bullet}, \Delta_{2}^{\bullet}$ and $\Delta_{3}^{\bullet}$. The implications for compact thermoacoustic sources are now explored.

\section{(i) Heat flux at a solid boundary}

When boundaries are present and $\Delta_{1}^{\bullet}$ is substituted in (5.7) the normal heat flux at the boundary, $q_{i} \hat{n}_{i}=q_{n}$ (positive into the fluid), leads to a surface monopole distribution of strength $\rho_{0}\left(\alpha / \rho c_{p}\right) q_{n}$ per unit area. This result holds for either fixed or moving boundaries. An oscillating heat flux $q_{n}$ on $\mathcal{S}$ is thus acoustically equivalent to vibrating an impermeable boundary with a normal velocity of $\left(\alpha / \rho c_{p}\right) q_{n}$, if terms in $q_{n}^{2}$ are neglected.

This source of sound has been discussed by Landau \& Lifshitz (1987) using matched expansions; by Howe $(1975, \S 8)$ by using volume sources in an acoustic analogy; and by Kempton $(1976, \S 2)$, who compared both these methods with a surface heat flux formulation. The examples discussed by these authors all relate to the small-amplitude case, with the solid boundary either an infinite plane surface, or an acoustically compact body. The results from all three methods are equivalent to the more general result stated here.

The small-amplitude restriction means $\Delta_{2}^{\bullet} \rightarrow 0$; while the other restrictions make it unnecessary to consider $\Delta_{3}^{\bullet}$, provided the thermal penetration depth in the fluid, $l_{\kappa}=\left(2 \kappa / \omega \rho c_{p}\right)^{1 / 2}$, is small in comparison with the acoustic wavelength $\lambda_{a c}=2 \pi c_{0} / \omega \dagger$. The radiated sound can then be expressed entirely in terms of the $\Delta_{1}^{\bullet}$ surface source distribution. Note that recognition of the inclusion of $\Delta_{1}^{\bullet}$ in the acoustic analogy removes one of Tam's objections to the latter as a description of aeroacoustic sources (2002, example 2).

The remainder of this section is concerned with mechanisms of sound generation where boundaries are not involved. The restriction to small-amplitude disturbances will be removed, allowing $\Delta_{2}^{\bullet}$ to become significant.

$\dagger$ In Howe (1998) §2.3.2 such variations are set equal to zero. An extreme case where this assumption fails is a bubbly liquid. For gases the assumption is reasonable.

$\dagger$ In the case of the plane boundary, use of the Neumann Green's function eliminates the contribution of normal dipoles placed on or close to the boundary. The same applies to any solid body whose radius of curvature is everywhere much greater than $l_{\kappa}$, given $l_{\kappa} \ll \lambda_{a c}$. 


\section{(ii) Unbounded flows: perfect gas}

In this case, $\alpha / \rho c_{p}=(\gamma-1) / \gamma P$, where $\gamma$ is the constant ratio of specific heats. From (5.2), $K_{e} / K_{0}=p^{2} / P P_{0}$, and either (5.1) or (5.3) lead directly to (5.9) for flows with $M^{2} \ll 1$. It follows that temperature gradients (as opposed to pressure gradients) contribute to $Q^{\star}$ only through the $\Delta_{1}^{\bullet}$ term in (5.9), and that

$$
\Delta_{1}^{\bullet}=-\frac{\partial}{\partial x_{i}}\left[\frac{\alpha}{\rho c_{p}} q_{i}\right] \approx \frac{\partial^{2}}{\partial x_{i}^{2}} F\left(T, P_{0}\right)
$$

where the error is a divergence term, of relative order $M^{2}$. Here

$$
\begin{aligned}
F\left(T, P_{0}\right) & =\int_{T_{0}}^{T}\left(\frac{\kappa \alpha}{\rho c_{p}}\right)_{\substack{T^{\prime}, P=P_{0} \\
\mathrm{~d} T^{\prime}}} \\
& =\frac{\gamma-1}{\gamma P_{0}} \int_{T_{0}}^{T} \kappa\left(T^{\prime}, P_{0}\right) \mathrm{d} T^{\prime}
\end{aligned}
$$

Thus

$$
\frac{\partial \Delta_{1}^{\bullet}}{\partial t} \approx\left(\frac{\gamma-1}{\gamma P_{0}}\right) \frac{\partial^{2}}{\partial x_{i}^{2}}\left[\kappa\left(T, P_{0}\right) \frac{\partial T}{\partial t}\right] .
$$

Equation (5.12) shows that in the absence of boundaries, temperature equilibration of hot spots in a heat-conducting perfect gas produces an equivalent source distribution that is linear in $\partial T / \partial t$, but is of quadrupole order. Combined with the $\left(\gamma P_{0}\right)^{-1}=\left(\rho_{0} c_{0}^{2}\right)^{-1}$ coefficient, which introduces an additional $M^{2}$ factor, this makes the radiation extremely weak even if the quadrupole strength

$$
\int \kappa\left(T, P_{0}\right) \frac{\partial T}{\partial t} \mathrm{~d} \mathcal{V}
$$

is non-zero when the integral is evaluated over the entire source region. $\dagger$

(iii) Unbounded flows: general fluid

Whenever the quantity

$$
\epsilon=\frac{\alpha}{\rho c_{p}}=\left(\frac{\partial V}{\partial h}\right)_{P}
$$

(where $h$ is the specific enthalpy and $V=\rho^{-1}$ ) is a function $\epsilon(P)$ of pressure alone, the arguments given in ( $i$ i) above for a perfect gas remain valid and there is no monopole source $\Delta_{2}^{\bullet}$, other than an $O\left(M^{2}\right)$ contribution from the pressure dependence of $\epsilon$. However if $\alpha / \rho c_{p}=\epsilon(T, P)$, with a significant temperature dependence at constant pressure (as in water, for example), (5.9) with $\mathrm{H}=1$ gives

$$
\Delta_{2}^{\bullet} \approx q_{i} \frac{\partial T}{\partial x_{i}} \epsilon_{T}=-\kappa \epsilon_{T}\left(\frac{\partial T}{\partial x_{i}}\right)^{2}, \quad\left(\text { where } \epsilon_{T}=\left(\frac{\partial \epsilon}{\partial T}\right)_{P}\right) .
$$

$\dagger$ Note, however, that in the small perturbation limit the temperature $T_{s}$ associated with the entropy mode obeys

$$
\frac{\partial T_{s}}{\partial t}=\nabla^{2}\left(\frac{\kappa_{0}}{\rho_{0} C_{p, 0}} T_{s}\right)
$$

so a linearised estimate of (5.13) leads to the conclusion that thermal diffusion in a perfect gas is at least a sextodecimopole (order 4) sound source. In fact its main effect is to cause attenuation of sound. 
This is a nonlinear contribution to $\Delta^{\bullet}$, whose instantaneous volume integral does not vanish. Fluctuations of this quantity (or, rather, its volume integral) in turbulent mixing will act as a monopole source of sound, analogous to fluctuations in the quantity $\Delta_{\mu}^{\bullet}$ that measures the rate of thermal expansion due to unsteady viscous dissipation (equation (5.4)).

Note that in a dilute (ideal) gas, $\epsilon=(\gamma-1) / \gamma P$ with $\gamma=\gamma(T)$, and in air the equilibrium value of $\gamma$ varies on account of the partially-excited vibrational degrees of freedom of $\mathrm{N}_{2}$ and $\mathrm{O}_{2}$. There is an issue - first raised by Kempton (1976) as to how far the equilibrium partial excitation of energy is maintained in air at audio frequencies. Thus although in principle the $\Delta_{2}^{\bullet}$ term acts as a monopole source of sound when hot and cold air mix in an unsteady manner, the situation is more complicated than the present analysis (based on equilibrium thermodynamics) indicates.

\section{(iv) Reacting and diffusing mixtures}

A monopole source term analogous to $\Delta_{2}^{\bullet}$ is also present in general when unsteady mixing occurs between two fluids of different composition; details are given in Morfey (1976). Note, however, that isothermal mixing of two different ideal gases is a special case, for which the monopole strength vanishes. A bursting helium balloon makes no noise unless it is under pressure!

\section{Conclusions}

\section{Appendix A. Second time-derivative of a windowed field variable}

The first step is to note that

$$
\frac{\partial^{2} \bar{\xi}}{\partial t^{2}}=\frac{\partial}{\partial t}[\xi \mathrm{H} \delta(t)]+\frac{\partial}{\partial t}\left[\Theta \frac{\partial}{\partial t}(\mathrm{H} \xi)\right] .
$$

The quantity $(\partial / \partial t)(\mathrm{H} \xi)$ can be rewritten in terms of the material derivative of $\xi$ by using the identity (2.7). Applying this to $\mathrm{H} \xi$ gives

$$
\begin{aligned}
\frac{\partial}{\partial t}(\mathrm{H} \xi) & =\frac{\mathrm{D}}{\mathrm{D} t}(\mathrm{H} \xi)-\frac{\partial}{\partial x_{i}}\left(\xi u_{i} \mathrm{H}\right)+\mathrm{H} \xi \Delta \\
& =\xi \frac{\mathrm{DH}}{\mathrm{D} t}-\frac{\partial}{\partial x_{i}}\left(\xi u_{i} \mathrm{H}\right)+\mathrm{H}\left(\frac{\mathrm{D} \xi}{\mathrm{D} t}+\xi \Delta\right)
\end{aligned}
$$

When (A 2) is substituted in (A 1) a term containing $(\partial / \partial t)\left(\xi u_{i} \mathrm{H}\right)$ appears. We can rewrite this by again using (2.7):

$$
\begin{aligned}
\frac{\partial}{\partial t}\left(\xi u_{i} \mathrm{H}\right) & =\frac{\mathrm{D}}{\mathrm{D} t}\left(\xi u_{i} \mathrm{H}\right)-\frac{\partial}{\partial x_{j}}\left(\xi u_{i} u_{j} \mathrm{H}\right)+\xi u_{i} \mathrm{H} \Delta \\
& =\xi u_{i} \frac{\mathrm{DH}}{\mathrm{D} t}-\frac{\partial}{\partial x_{j}}\left(\xi u_{i} u_{j} \mathrm{H}\right)+\mathrm{H}\left(\frac{\mathrm{D} \xi}{\mathrm{D} t} u_{i}+\xi \frac{\mathrm{D} u_{i}}{\mathrm{D} t}+\xi u_{i} \Delta\right) .
\end{aligned}
$$


We therefore have

$$
\begin{aligned}
\frac{\partial^{2} \bar{\xi}}{\partial t^{2}}= & \frac{\partial}{\partial t}[\xi \mathrm{H} \delta(t)]-\frac{\partial}{\partial x_{i}}\left[\xi u_{i} \mathrm{H} \delta(t)\right]+\frac{\partial}{\partial t}\left(\xi \frac{\mathrm{DH}}{\mathrm{D} t} \Theta\right)-\frac{\partial}{\partial x_{i}}\left(\xi u_{i} \frac{\mathrm{DH}}{\mathrm{D} t} \Theta\right) \\
& +\frac{\partial}{\partial t}\left(\frac{\mathrm{D} \xi}{\mathrm{D} t}+\xi \Delta\right)-\frac{\partial}{\partial x_{i}}\left[\overline{\xi \frac{\mathrm{D} u_{i}}{\mathrm{D} t}+u_{i}\left(\frac{\mathrm{D} \xi}{\mathrm{D} t}+\xi \Delta\right)}\right]+\frac{\partial^{2}}{\partial x_{i} \partial x_{j}}\left(\overline{\xi u_{i} u_{j}}\right) .
\end{aligned}
$$

\section{Appendix B. Generalized Lilley-Goldstein equation}

(a) The exact Goldstein analogy for an ideal fluid

Goldstein (2001) produced an exact acoustic analogy equation that is more general than (3.20), in that the base flow is a parallel, steady, streamwise-uniform shear flow:

$$
\tilde{u}_{i}=U\left(x_{2}, x_{3}\right) \delta_{1 i}, \quad \tilde{\rho}=\tilde{\rho}\left(x_{2}, x_{3}\right), \quad \tilde{c}=\tilde{c}\left(x_{2}, x_{3}\right) .
$$

Here a tilde denotes base-flow variablest, and $x_{1}$ is the streamwise direction. Goldstein's equation has the form

$$
\mathcal{L}(\pi)=Q
$$

in which $\pi$ (defined below) is a wave variable related to pressure, $\mathcal{L}$ is a modified version of the Lilley-Goldstein convected wave operator (Lilley 1974, Goldstein 1976, Tester \& Morfey 1976), and $Q$ is a source term that is nonlinear in the quantities

$$
u_{i}^{\prime}=u_{i}-\tilde{u}_{i}, \quad\left(c^{2}\right)^{\prime}=c^{2}-\tilde{c}^{2}, \quad \text { and } \pi .
$$

The advantage of Goldstein's equation is that it is able to model both refraction in strongly-sheared flows, and amplification of aerodynamic sound by velocity and density gradients in the source region (Balsa 1977, Kempton 1977, Tester \& Morfey 1976), as consequences of the linear wave operator $\mathcal{L}$ (rather than via additional source terms $Q$ ). At the same time it is exact within the limitations of the fluid model used, which are:

1. The isentropic compressibility $1 / \rho c^{2}=K$ is a function $K(P)$ of the pressure alone, i.e. the fluid is barotropic with respect to $K$.

2. The fluid is inviscid and non-conducting.

Here we aim to show how Goldstein's source term $Q$ relates to the source identified in $(3.20)$ as

$$
-\frac{\partial}{\partial x_{i}}\left[\frac{\left(\rho-\rho^{\star}\right)}{\rho} \frac{\partial p}{\partial x_{i}}\right]=-\frac{\partial F_{i}}{\partial x_{i}}, \quad \text { say, }
$$

where $F_{i}$ is an equivalent body force (per unit volume) applied to a uniform ideal fluid at rest. The corresponding term in $Q$ (Goldstein 2001) is

$$
-\frac{\tilde{\mathrm{D}}}{\mathrm{D} t}\left(\frac{\partial \hat{f}_{i}}{\partial x_{i}}\right)+2 \frac{\partial}{\partial x_{1}}\left(\hat{f}_{j} \frac{\partial U}{\partial x_{j}}\right), \quad \hat{f}_{i}=-\left(c^{2}\right)^{\prime} \frac{\partial \pi}{\partial x_{i}}
$$

$\dagger$ Overbars were used in Goldstein (2001) but could here be confused with windowed quantities.

$\ddagger$ Goldstein (2001) actually assumed the fluid to be a perfect gas, which is a special case of limitation 1.

ๆ A sign error in Goldstein (2001) has been corrected 
here $\hat{f}_{i}$ is an equivalent body force (per unit mass) applied to the base flow, and $\tilde{\mathrm{D}} / \mathrm{D} t \equiv \partial / \partial t+U \partial / \partial x_{1}$. The $\hat{f}_{i}$ expression in (B 4) refers to a perfect gas, but can be generalized to the barotropic-fluid model of limitation 1 as follows.

(b) Generalized form of Goldstein's analogy for a barotropic fluid

Given that

$$
\left.\frac{1}{\rho c^{2}} \equiv \frac{\partial \ln \rho}{\partial P}\right|_{s}=K(P)
$$

we define the isentropic density exponent $\theta$ and the isentropic density $\rho^{\diamond}$ as

$$
\theta=\int_{P_{0}}^{P} K\left(P^{\prime}\right) \mathrm{d} P^{\prime}=\theta\left(P ; P_{0}\right), \quad \rho^{\diamond}=\rho_{0} \mathrm{e}^{\theta}=\rho^{\diamond}\left(P ; P_{0}, \rho_{0}\right) ;
$$

here $\rho_{0}, P_{0}$ are constant reference values. The continuity and momentum equations for an ideal fluid described by (B 5), subject to a body force $g_{i}$ per unit mass, are

$$
\frac{\partial u_{i}}{\partial x_{i}}=-\frac{1}{\rho^{\diamond}} \frac{\mathrm{D} \rho^{\diamond}}{\mathrm{D} t}, \quad \rho^{\diamond} \frac{\mathrm{D} u_{j}}{\mathrm{D} t}=-c^{2} \frac{\partial \rho^{\diamond}}{\partial x_{j}}+\rho^{\diamond} g_{j}
$$

where $\mathrm{D} / \mathrm{D} t \equiv \partial / \partial t+u_{i} \partial / \partial x_{i}$; the usual fluid dynamic variables $P, \rho$ are replaced in (B 7) by $\rho^{\diamond}, c^{2}$. These equations are exact, but can nevertheless be reduced to the same form as the linearized Euler equations with an applied body force $f_{i}$ by following the steps in Goldstein (2001). The result, expressed in terms of the 'perturbation variables'

$$
\frac{\rho^{\diamond}}{\rho_{0}} u_{i}^{\prime}=m_{i} \quad \text { and } \quad \frac{\rho^{\diamond}}{\rho_{0}}-1=\pi
$$

(where $\pi$ corresponds to Goldstein's $\pi$ defined for a perfect gas), is

$$
\frac{\tilde{\mathrm{D}} \pi}{\mathrm{D} t}+\frac{\partial m_{i}}{\partial x_{i}}=0, \quad \frac{\tilde{\mathrm{D}} m_{j}}{\mathrm{D} t}+m_{i} \frac{\partial \tilde{u}_{j}}{\partial x_{i}}+\tilde{c}^{2} \frac{\partial \pi}{\partial x_{j}}=f_{j}
$$

with the applied body force $f_{j}$ given by

$$
f_{j}=(1+\pi) g_{j}-\left(c^{2}\right)^{\prime} \frac{\partial \pi}{\partial x_{j}}-\frac{\partial}{\partial x_{i}}\left[(1+\pi) u_{i}^{\prime} u_{j}^{\prime}\right] .
$$

The second term on the right is the $\hat{f}_{j}$ 'temperature dipole' found by Goldstein (2001) and given in (B 4). Its equivalence to a body force driving small-amplitude perturbations to the base flow is seen by comparing it with the first term.

It is straightforward to eliminate $m_{i}$ from (B 9) to obtain a Lilley-Goldstein equation that is exact, given assumptions $1 \& 2$ above. Since $\tilde{\rho} \tilde{c}^{2}$ is a constant throughout the base flow, the $\pi$ variable of (B 8) can be exchanged for a pressurelike variable,

$$
p^{+}=\tilde{\rho} \tilde{c}^{2} \pi=\tilde{\rho} \tilde{c}^{2}\left(\mathrm{e}^{\theta}-1\right) .
$$

In a region of small-amplitude pressure disturbances, where $\left(p / \tilde{\rho} \tilde{c}^{2}\right)=\zeta \ll 1$, $p^{+} \approx p$. Specifically

$$
p^{+}=p\left[1+(\beta-1) \zeta+O\left(\zeta^{2}\right)\right]
$$


where $\beta$ is the coefficient of nonlinearity $c^{-1}(\partial(\rho c) / \partial \rho)_{s}=\frac{1}{2}\left[1-K^{\prime}(P) / K^{2}(P)\right]$, evaluated at $\tilde{P}=P_{0}$. The exact shear-flow analogy in terms of $p^{+}$is

$$
\mathcal{L}\left(p^{+}\right)=\tilde{\rho} Q, \quad Q=-\frac{\tilde{\mathrm{D}}}{\mathrm{D} t}\left(\frac{\partial f_{i}}{\partial x_{i}}\right)+2 \frac{\partial}{\partial x_{1}}\left(f_{j} \frac{\partial U}{\partial x_{j}}\right)
$$

where $\mathcal{L}$ is the Lilley-Goldstein operator:

$$
\mathcal{L} \equiv \frac{\tilde{\mathrm{D}}}{\mathrm{D} t}\left[\frac{1}{\tilde{c}^{2}} \frac{\tilde{\mathrm{D}}^{2}}{\mathrm{D} t^{2}}-\tilde{\rho} \frac{\partial}{\partial x_{i}}\left(\frac{1}{\tilde{\rho}} \frac{\partial}{\partial x_{i}}\right)\right]+2 \frac{\partial}{\partial x_{1}}\left(\frac{\partial U}{\partial x_{j}} \frac{\partial}{\partial x_{j}}\right)
$$

The operator $\mathcal{L}$ is given here in the generalized form introduced by Tester \& Morfey (1976) for small perturbations to an arbitrary fluid in parallel shear flow; for 2D or axisymmetric base flows it reduces to the Pridmore-Brown operator (in $x_{2}$ or $r$ respectively), on Fourier transformation with respect to the other independent variables.

(c) Comparison of dipole terms in Goldstein and Lighthill analogies

We now compare the $\left(c^{2}\right)^{\prime}$ term in (B 10), i.e. the equivalent body force per unit mass

$$
\hat{f}_{i}=-\left(c^{2}\right)^{\prime} \frac{\partial \pi}{\partial x_{i}}
$$

with its counterpart from (B 3), namely the force per unit volume

$$
F_{i}=\frac{\left(\rho-\rho^{\star}\right)}{\rho} \frac{\partial p}{\partial x_{i}} .
$$

The squared sound speed in (B 15) is related to the isentropic compressibility by

$$
c^{2}=\frac{1}{K(P)} \frac{1}{\rho} \text {. }
$$

Because $K$ is assumed to depend only on $P$ (unlike $\rho$ ), we can expand $c^{2}(K, \rho)$ in powers of $\alpha=K / \tilde{K}-1$, where $\tilde{K}$ is the base-flow compressibility: thus

$$
\begin{aligned}
\left(c^{2}\right)^{\prime} & =\frac{1}{(1+\alpha) \tilde{K} \rho}-\frac{1}{\tilde{K} \tilde{\rho}} \\
& =\frac{1}{\tilde{K}}(V-\tilde{V}-\alpha V)\left[1+O\left(\alpha^{2}\right)\right], \quad(\alpha \sim \zeta \ll 1) .
\end{aligned}
$$

Here $V=1 / \rho$ denotes the specific volume of the fluid. The gradient of $\pi$ in (B 15) is related to the gradient of $p$ via (B 11, B 12); also $\alpha$ is related to $\zeta$ by

$$
\alpha=(1-2 \beta) \zeta+O\left(\zeta^{2}\right)
$$

for the present fluid model (B 5). Combining these results shows that Goldstein's $\hat{f}_{i}$ temperature dipole in (B 15) is equivalent to an applied force per unit volume

$$
\tilde{\rho} \hat{f}_{i}=\frac{1}{\rho} \frac{\partial p}{\partial x_{i}}\left\{\varrho_{e}-2(\beta-1) \rho\left[\zeta+O\left(\zeta^{2}\right)\right]\right\},
$$


with $\varrho_{e}=\rho-\tilde{\rho}-p / \tilde{c}^{2}$ defined by analogy with (B 16).

Even when the base flow is reduced to a uniform fluid at rest, i.e.

$$
\tilde{\rho} \rightarrow \rho_{0}(=\text { const. }), \quad \tilde{c} \rightarrow c_{0}(=\text { const. }), \quad \tilde{u}_{i} \rightarrow 0, \quad-\mathcal{L} \rightarrow \frac{\partial}{\partial t} \square^{2},
$$

minor differences remain between the equivalent body force (B 20) in the exact Goldstein analogy, and (B 16) obtained via the Lighthill analogy in $\S ? ?$. A possible reason is the difference in wave variable, although $p^{+} \approx p$ in the acoustic far field. In addition, the Goldstein source terms in (B 13) lack an explicit monopole component corresponding to $Q$ in (3.20) and (5.3). The latter expression reduces, under the same conditions assumed in deriving the Goldstein analogy, to the nonlinear monopole

$$
Q^{\mathrm{NL}}=(\beta-1) \rho_{0} K_{0}^{2} \frac{\mathrm{D} p^{2}}{\mathrm{D} t}[1+O(\zeta)]
$$

whereas the Goldstein body force in (B 20) exceeds the Lighthill-analogy version (B 16) by a nonlinear contribution

$$
F_{i}^{\mathrm{NL}}=-(\beta-1) K_{0} \frac{\partial p^{2}}{\partial x_{i}}[1+O(\zeta)]
$$

when (B 21) is applied. Since the corresponding source terms in $\square^{2} p$ (or $\square^{2} p^{+}$) = $-q$ are $\partial Q^{\mathrm{NL}} / \partial t$ (Lighthill) and $-\partial F_{i}^{\mathrm{NL}} / \partial x_{i}$ (Goldstein), the difference to leading order is

$$
\frac{\partial Q^{\mathrm{NL}}}{\partial t}+\frac{\partial F_{i}^{\mathrm{NL}}}{\partial x_{i}} \approx \frac{\beta-1}{\rho_{0} c_{0}^{2}} \square^{2} p^{2} \approx \square^{2}\left(p^{+}-p\right) .
$$

Equation (B 24) shows that the apparent discrepancy between the dipole terms in the two analogies is counteracted by an additional monopole term in the Lighthill formulation, leaving a residual source term that represents the near-field difference between $p^{+}$and $p$ as in (B 12). Thus for purposes of far-field radiation the Goldstein body force (B 15) effectively combines the dipole and monopole source terms given by (3.20), given that the fluid is ideal with $K=K(P)$.

M. C. M. Wright was supported by an EPSRC Advanced Research Fellowship.

\section{References}

Bailly, C. \& Bogey, C. 2004 Contributions of computational aeroacoustics to jet noise research and prediction. Int. J. Comp. Fluid Dyn. 18 481-491. (doi:10.1080/10618560410001673498)

Balsa, T. F. 1977 The acoustic field of sources in shear flow with application to jet noise: convective amplification. J. Fluid Mech. 79, 33-47.

Brentner, K. S. \& Farassat, F. 1998 An analytical comparison of the acoustic analogy and Kirchhoff formulation for moving surfaces. AIAA J. 36, 1379-1386.

Colonius, T. \& Lele, S. K. 2004 Computational aeroacoustics: progress on nonlinear problems of sound generation. Prog. Aerospace Sci. 40, 345-416. (doi:10.1016/j.paerosci.2004.09.001)

Curle, N. 1955 The influence of solid boundaries upon aerodynamic sound. Proc. Roy. Soc. Lond. A 231, 505-514. 
Doak, P. E. 1960 Acoustic radiation from a turbulent fluid containing foreign bodies. Proc. Roy. Soc. Lond. A 254, 129-145.

Farassat, F. \& Myers, M. K. 1988 Extension of Kirchhoff's formula to radiation from moving surfaces. J. Sound Vib. 123, 451-460.

Farassat, F. \& Myers, M. K. 1990 An analysis of the quadrupole noise source of high speed rotating blades. In Computational Acoustics Vol. 2 (ed. D. Lee, A. Cakmak \& R. Vichnevetsky), pp. 227-240. Elsevier Science Publishers B.V.

Ffowcs Williams, J. E. 1969 Hydrodynamic noise. Ann. Rev. Fluid Mech. 1, 197-222. (doi:10.1146/annurev.fl.01.010169.001213)

Ffowcs Williams, J. E. \& Hawkings, D. L. 1969 Sound generation by turbulence and surfaces in arbitrary motion. Phil. Trans. Roy. Soc. A 264, 321-342.

Goldstein, M. E. 1976 Aeroacoustics, $\S \S 1.2,6.6$. New York: McGraw-Hill.

Goldstein, M. E. 2001 An exact form of Lilley's equation with a velocity quadrupole/temperature dipole source term, J. Fluid. Mech. 443, 231-236.

Goldstein, M. E. 2002 A unified approach to some recent developments in jet noise theory. Int. J. Aeroacoust. 1, 1-16. (doi:10.1260/1475472021502640)

Goldstein, M. E. 2003 A generalized acoustic analogy. J. Fluid Mech. 488, 315-333. (doi:10.1017/S0022112003004890)

Goldstein, M. E. 2005 On identifying the true sources of aerodynamic sound. J. Fluid Mech. 526, 337-347. (doi:10.1017/S0022112004002885)

Hamilton, M. F. \& Morfey, C. L. 1997 Model equations, in Nonlinear Acoustics, (ed. M. F. Hamilton \& D. T. Blackstock), pp. 41-56. San Diego: Academic Press.

Howe, M. S. 1975 Contributions to the theory of aerodynamic sound, with application to excess jet noise and the theory of the flute. J. Fluid. Mech. 71, 625-673.

Howe, M. S. 1998 Acoustics of Fluid-Structure Interactions. Monographs on Mechanics. Cambridge University Press.

Hu, Z., Morfey, C. L. \& Sandham, N. D. 2003 Sound radiation in turbulent channel flows. J. Fluid Mech. 475, 269-302. (doi:10.1017/S002211200200277X)

Kambe, T. 1984 Influence of viscosity on aerodynamic sound emission in free space. $J$. Sound Vib. 95, 351-360. (doi:10.1016/0022-460X(84)90674-6)

Kambe, T. \& Minota, T. 1983 Acoustic wave radiated by head-on collsion of two vortex rings. Proc. R. Soc. Lond. A 386, 277-308.

Kempton, A. J. 1976 Heat diffusion as a source of aerodynamic sound. J. Fluid Mech. 78, $1-31$.

Kempton, A. J. 1977 Acoustic scattering by density gradients. J. Fluid Mech. 83, 495-508.

Landau, L. D. \& Lifshitz, E. M. 1987 Fluid Mechanics, §74, Problem 9, 2nd edn. Oxford: Pergamon.

Lilley, G. M. 1974 On the noise from jets. Ch. 13, Noise Mechanisms AGARD-CP-131 pp. 13.1-13.12.

Lilley, G. M. 1996 The radiated noise from isotropic turbulence with applications to the theory of jet noise. J. Sound Vib. 190, 463-476. (doi:10.1006/jsvi.1996.0074)

Lighthill, M. J. 1952 On sound generated aerodynamically. I. General theory. Proc. R. Soc. Lond. A 211, 564-587.

Lighthill, M. J. 1978 Waves in Fluids. Cambridge: Cambridge University Press.

Morfey, C. L. 1973 Amplification of aerodynamic noise by convected flow inhomogeneities. J. Sound Vib. 31, 391-397.

Morfey, C. L. 1976 Sound radiation due to unsteady dissipation in turbulent flows. $J$. Sound Vib. 48, 95-111. (doi:10.1016/0022-460X(76)90374-6)

Morgans, W. R. 1930 The Kirchhoff formula extended to a moving surface. Phil. Mag. 9, $141-161$. 
Morse, P. M. \& Feshbach, H. 1953 Methods of Theoretical Physics: Part I, §7.3. New York: McGraw-Hill.

Obermeier, F. 1975 Sound generation by heated subsonic jets. J. Sound Vib. 41, 463-472.

Obermeier, F. 1985 Aerodynamic sound generation by viscous processes. J. Sound Vib. 99, 111-120. (doi:10.1016/0022-460X(85)90448-1)

Powell, A. 1960 Aerodynamic noise and the plane boundary. J. Acoust. Soc. Am. 32, 982-990. (doi:10.1121/1.1908347)

Rayleigh, Lord 1894 Theory of Sound, §196. London: Macmillan.

Shur, M. L., Spalart, P. R. \& Strelets, M. K. 2005 Noise prediction for increasingly complex jets. Part I: Methods and tests. Int. J. Aeroacoustics 4, 213-246. (doi:10.1260/1475472054771376)

Singer, B. A., Brentner, K. S., Lockard, D. P. \& Lilley, G. M. 2000 Simulation of acoustic scattering from a trailing edge J. Sound Vib. 230, 541-560. (doi:10.1006/jsvi.1999.2628)

Spalart, P., Shur, M., Wright, M. C. M. \& Morfey, C. L. 2007 Variants of the Ffowcs Williams-Hawkings equation with favourable properties for coupling with turbulence simulations J. Fluid Mech. Submitted

Tam, C. K. W. 2002 Computational aeroacoustics examples showing the failure of the acoustic analogy theory to identify the correct noise sources. J. Comp. Acoust. 10, 387-405. (doi:10.1142/S0218396X02001607)

Tester, B. J. \& Morfey C. L. 1976 Developments in jet noise modelling - theoretical predictions and comparisons with measured data. J. Sound Vib. 46, 79-103. (doi:10.1016/0022-460X(76)90819-1) 